\title{
The Design and Implementation of Visual Intelligent Doorbell System
} Xiao LIU ${ }^{1, a}$,Fensu SHI ${ }^{1, b}$, Pan SHI ${ }^{1}$,Zicong $\mathrm{LI}^{1}$

${ }^{1}$ Department of Computer Science and Engineering, Beifang University of Nationalities, Yinchuan, Ningxia, 750021, China

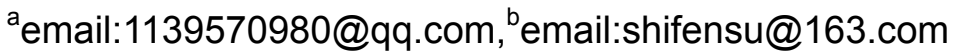

\section{Keywords: Intelligent Doorbell System; Embedded, Remote Video Monitoring}

\begin{abstract}
Based on actual demand of the bell system, this visual intelligent doorbell system was designed and implemented to be a high reliability, good performance and low cost embedded bell system combined with the embedded technology, image processing technology, image transmission technology, Zigbee technology and Web server technology. The system selects the S3C6410 microprocessor as the core controller, using USB camera to capture visitors' video, realizing the SMS communication function with mobile terminal by using GPRS communication technology, achieve the acquisition and wireless transmission of the temperature and humidity by using Zigbee technology, and realizing reliable remote video monitoring through the embedded Web server technology.
\end{abstract}

\section{Introduction}

With the rapid development of domestic economy, the intelligent construction has been carried out rapidly. and as an important part of intelligent household, visual doorbell system occupies an important position of intelligent community construction, it is the guarantee for safety. On the other hand, as the internet has been influenced people's daily life, entertainment and all aspects of the work deeply, in order to satisfy the consumers' demand of the bell system about security, intelligence, information demand, network, miniaturization, etc. the visual doorbell system use the embedded technology, network communication technology and linux technology comprehensively. We can foresee digital audio and video processing technology will be the future development and application trends of the security systems.

\section{Overall system design}

The visual doorbell system is mainly used in residential quarters, villas, office buildings and other places. Combining the characteristics that the embedded technology supporting the TCP/IP protocol, and using advanced network technology achieve the transmission of video and control signal and the network interconnection and access through establishing the user server.

\section{Function module design}

The function module of this visual doorbell system mainly includes: (1) the video acquisition module, the camera real-time acquire and transmit the video for video monitoring area[1][2][3];(2) the remote video monitoring module, the user can real-time monitor the video through logining the Web server[4]; (3) the GPRS communication module, achieve the message communication with mobile phone terminal[5]; (4) the temperature and humidity acquisition module, the acquisition and real-time display of the outdoor temperature and humidity[6]; (5) Mp3 ringtones module, implementation time and date's display and colorful Mp3 remingding when on dining time and temperature and humidity out of range of set value. 


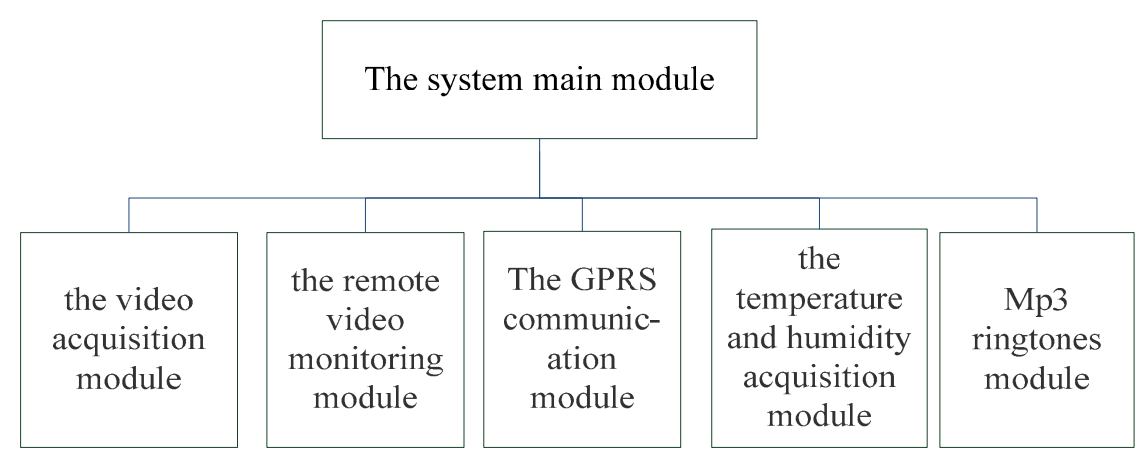

Figure 1 system function module

\section{Hardware architecture design}

1. the system master control unit. S3C6410 demoboard which take S3C6410 microprocessor as the core, the main configuration include FLASH, SDROM, touch screen, USB interface, network interfaces, RS-232 serial port, and audio interface. it also includes many powerful hardware accelerator, an integration of multiple formats codec (MFC) supporting MPEG4/h.263/h.264decoding.

2. the ZigBee wireless module. Adopting TI CC2431 chip, built-in hardware engine positioning and enhanced 8-bit 51 single chip microcomputer, IEEE802.15.4 / ZigBee standard specification, frequency range of $2045 \mathrm{M}$ to $2483.5 \mathrm{M}$, communication distance at around 30 meters, has a piece of $64 \mathrm{k}$ programmable flash, and $8 \mathrm{k}$ RAM.

3. the camera. Selection of stellar micro ZC301 digital camera.

4. the GPRS module. Using SIM900A module. SIM900A is a 2 frequency of GSM/GPRS module, frequency at EGSM $900 \mathrm{MHZ}$ and $1800 \mathrm{MHZ}$ DCS. the extension of the TCP/IP AT commands make the user use TCP/IP protocol easily.

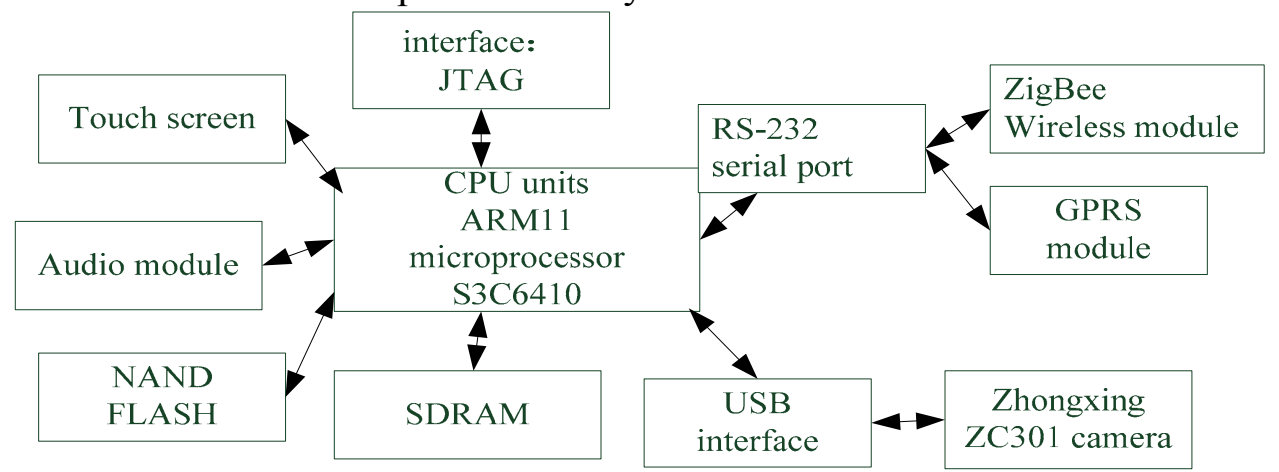

Figure 2 System hardware structure.

\section{The implementation of system main function module}

UP-CUP6410 platform is the target platform for this system, Emebedded linux kernel 3.6.5 running on it. The host PC running Ubuntu Linux operating system through using the virtual machine (VMware Workstation)under the Windows 7 operating system. Install the ARM-Linux cross compile environment ARM-Linux- GCC3.4.6 and c ++ graphical user interface Qt 4.4.0. IAR Embeddded Workbench for MCS-51 is been used to programme ZigBee wireless module.

\section{Video acquisition module}

video acquisition module is the key part of the visual doorbell system, this modules realize the image acquisition of the visitors through camera.

In linux camera driver using spca5xx driver, so you need to transplant the camera driver. after that the video acquisition can been programmed using Video4Linux. The Video4Linux (V4L) is the kernel driver of video equipment in linux, it provides a series of interface functions for video equipment application programming. 


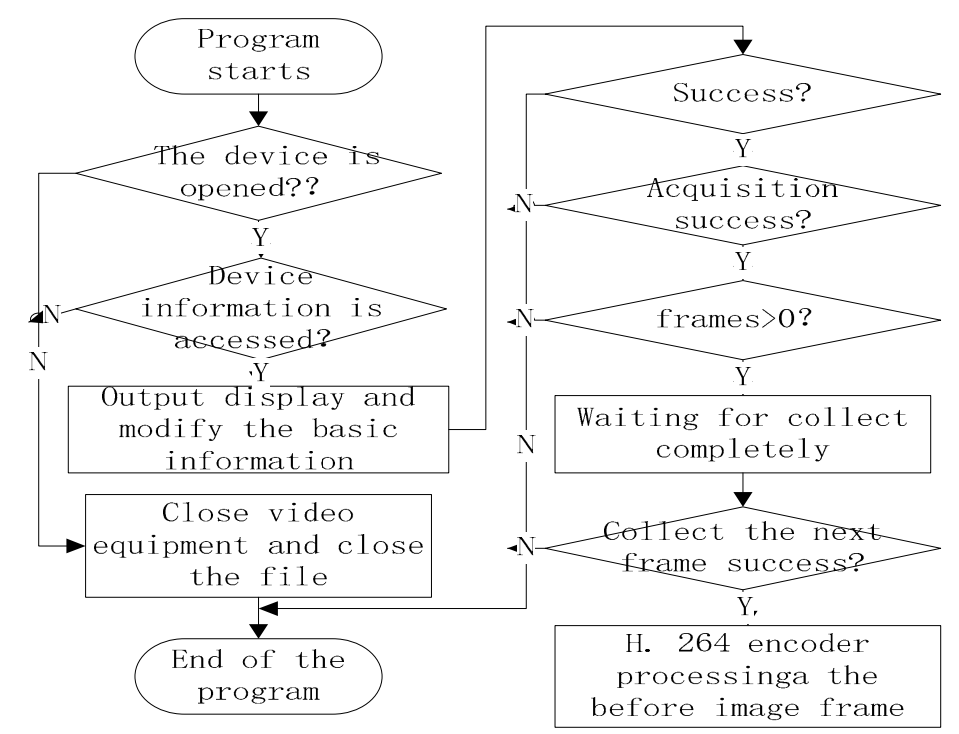

figure 3 flow chart of video acquisition

\section{GPRS communication module}

GPRS network support TCP/IP protocol, which makes much easier on the wireless data transmission. The purpose of design GPRS communication module is implementation the SMS communication function with mobile terminal using real-time multitasking embedded Linux operating system and graphical development environment QT.

The AT command is the agreement language of the communication which between demoboard and the GPRS module. GPRS communication system using the AT command to send control commands to the GPRS module through a serial port.

GPRS module send SMS requires two steps: first sends the AT + CMGS, make the GPRS module into the state of waiting to send information, and then send AT primitive code.

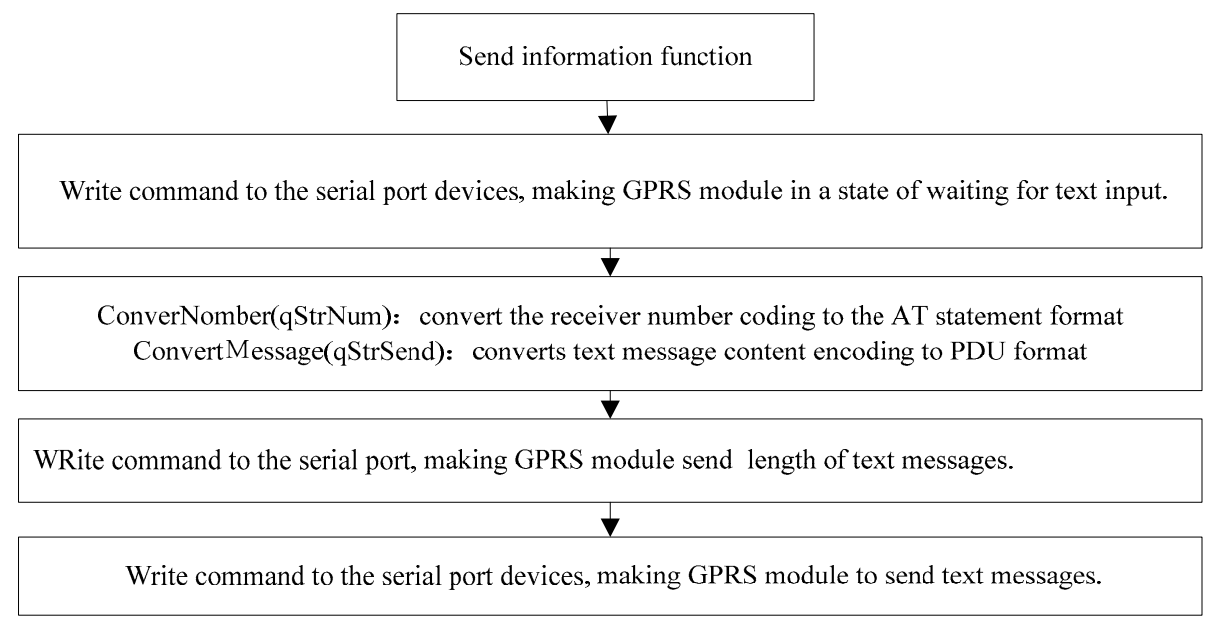

\section{figure 4 The flow chart of SMS messages}

When a text message came, GPRS module will send signal"RING" to the serial port. Receive a text messages, need to convert the message primitives decoding to the normal format which the system can identify.

Convert the SMS primitive decoding into information, including the phone number, the time of send messages, the message content and so on.

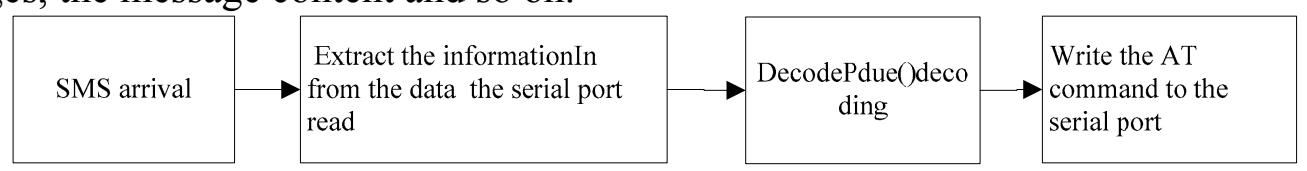

Figure 5 the flow chart of SMS receiving 


\section{Remote monitoring module}

This module implements an embedded remote monitoring system of the Web, which based on $\mathrm{B} / \mathrm{S}$ architecture. Clients use ordinary PC browser connection the network, then access the IP address of demoboard to login monitoring system to monitor the operation. The Web remote monitoring module, divided into the following three child module: Embedded Web server module, CGI universal gateway module, video server module.

The function diagram of module is shown in figure 6.

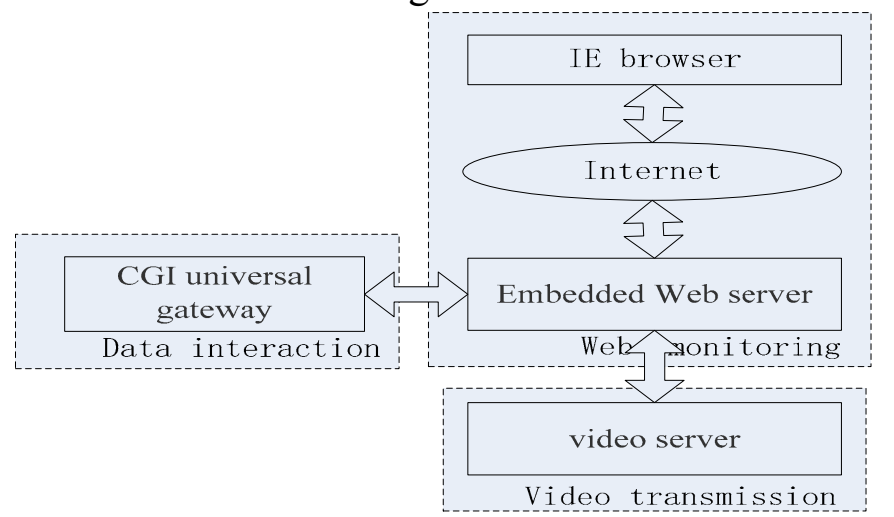

1.the embedded Web server Boa

Figure 6 module partition of remote monitoring system

The process of Boa server work is mainly includes: the Web server's initialization work; After receiving the connection requests of the client browser, make corresponding processing according to the request; when web server processing finished, sends response information to the client browser and close the TCP connection with client.

The steps of transplantation boa server:

(1) Download the boa-0.94.13. Tar. gz source code and decompression.

(2) Run the configure command under the SRC directory, modify the generated makefile and compile it. And downloaded the boa executable file to the demoboard.

(3) Modify the Boa server configuration file according to the actual demand, boa.conf. Change is completed, the download/etc/departure board boa directory.

(4) Run the boa file under the /SRC directory what extracted from the boa.0.94.13 - tar. gz directory.

(5) Add the boa files to the file system, download the file system again, so that the system would automatically run the boa service when starting build /WWW directory.

(6) Test and check Boa server

Enter the IP address of the demoboard in PC browser, test page is displayed properly shows that Boa transplant success.

2. servfox video server

This system has realized when enter the IP address of the video server to the client PC browser which networking in the local area network (LAN), the surveillance video will be displayed on the web site. Servfox is the video server. Servfox is the simplification version of spcaview package, spcaview was a open-source software packages to implement the network video server under Linux system.

Servfox video server transplant:

(1) UnZip servfox-R1 j_3.tar.gz。

(2) Modify Makefile.arm and renamed to Makefile.

Modify CC=arm-linux-gec to CC=/opt/a:osstools/gec-3.4.6-glibc-2.3.6/bin/ann-linux

Compiled, then download servfox executable file to demoboard/MNT/yaffs.

3.CGI gateway submodule

CGI technology, named Common Gateway Interface, is an standard Interface which the external extension application and the embedded Web server interacted in. Its working process is: when user access to the browser, a request will pointing to a URL of CGI application via an HTML form or hyperlinks, embedded Web server parse arguments after receiving the request, and perform the 
corresponding CGI application, then CGI application format the execution result to HTML document, Then the embedded Web server processing the data content that the CGI application returned according to the data head, and transmitted to the client browser, then displayed by the client browser.

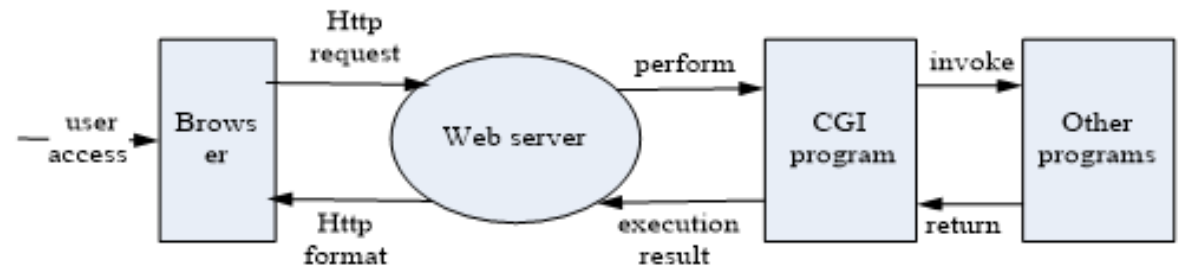

Figure 7 the flow of CGI work

4. the realization of embedded Web video server

(1) transplant servfox

(2) loading ZC0301 camera driver, start the Boa server, perform servfox executable file [/ MNT/yaffs]. /dev/video/servfox - g - d /dev/video - s 640 x480-w7070

(3) run servfox video monitoring based on $\mathrm{B} / \mathrm{s}$ structure

Transplate the directory http- Java -applet in spcaview package to Boa's default directory /var/WWW in demoboard, and set permissions of this file.

Input the address of demoboard in PC browser to start the embedded Web server.

\section{temperature and humidity real-time display module}

When the temperature and humidity is beyond the scope of rated, the system will play MP3 music .so does the repast time.

As a new wireless technology, the basis of ZigBee is a standard known as IEEE802.15.4 technical standards. Mainly used for short distance and low power consumption and low transmission rate data transmission between various kinds of electronic equipment.

This system use the Zigbee technology to realize the collection of temperature and humidity and the response of the system time.

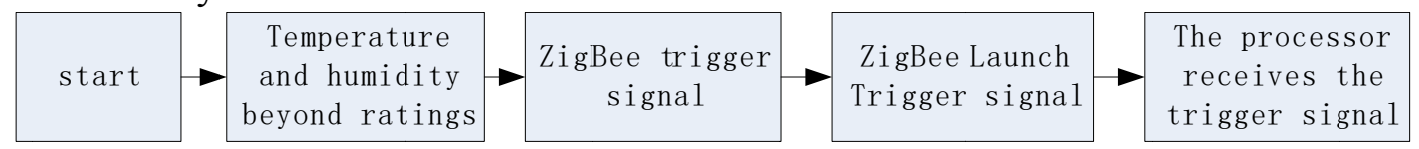

Figure 8 ZigBee work flow chart

\section{System testing}

After a series of design and debugging of software and hardware, the design of intelligent visual doorbell system based on S3C6410 is completed, and achieve the established goals well. Specific debugging result is shown below.

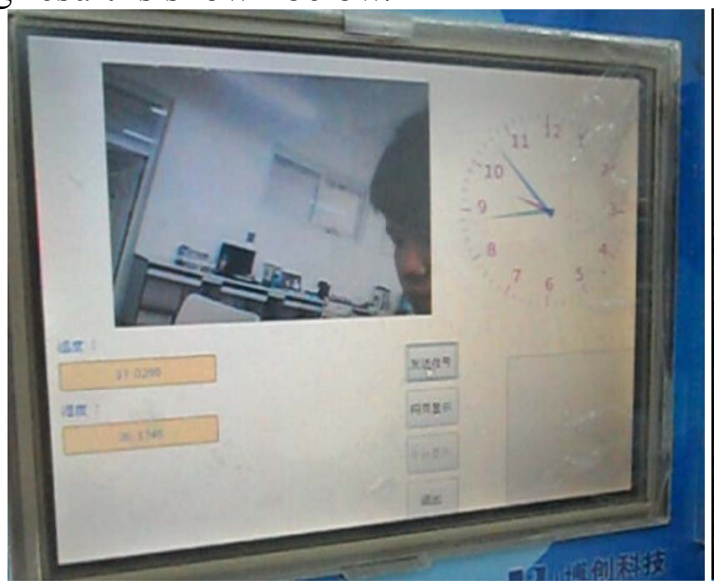

Figure 9 intelligent doorbell system user

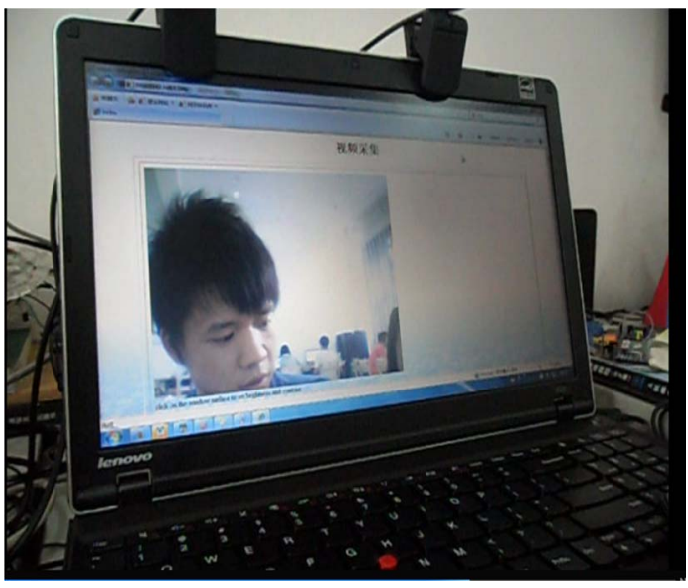

Figure 10 video remote monitoring 
interface andvideo collection figure

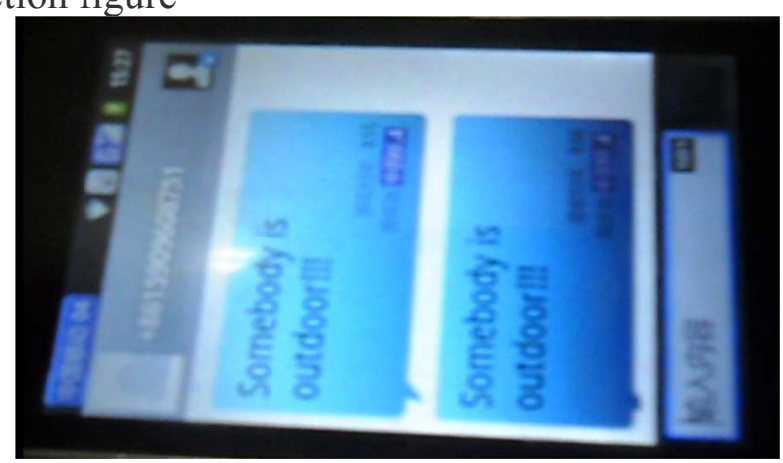

Figure 11 GPRS SMS communication

\section{Summary}

This system uses a modular design method, completed the expected functions of intelligent doorbell system, along with the computer technology, multimedia technology, network technology and the rapid development of smart home, intelligent household based on embedded will tend to be more humanized, comprehensive, digital and networked, this intelligent doorbell system as a part of the smart home will provide a research direction for wider and profoundly research of smart home in future.

\section{Acknowledgment}

This work is supported by National Natural Science Foundation of China (161162013, 61461003), the graduate innovation project of 2014 Beifang University of Nationalities and a project supported by National Natural Science Foundation pre-breeding program of Beifang University of Nationalities (No.2012QZP01).

\section{References}

[1] Juguangt Li. ARM Application system development explanation. Peking: Tsinghua University Press, 2003,1 15

[2] Jili Ni. QT and LINUX operating system window design [M].Peking: Electronic Industry Press, 2006.4

[3] Shiyu Long, Wu Wei, Biyan Lu. The design of embedded video monitoring system based on ARM [J]. Journal of electronic design engineering, 2011, 19 (17) : 136, 139.

[4] KaiSong, Liping Yan, LanGan. The design and implementation of embedded Web server [J]. Computer Engineering and Design,2009(004):808-810

[5] Xiaohai Pei. Research and design of GPRS communication system based on ARM [D], Sichuan University,2010

[6] Qiaoyun Guo, Xuemei $\mathrm{Xu}$, The design of the wireless video monitoring system based on ARM11 [J], physics institute of Central south university,2009,20-45 